\title{
Trayectos de uso de TIC: Caso de la Universidad Javeriana
}

\section{Resumen}

El propósito de la investigación fue conocer de qué manera los docentes universitarios de la Pontificia Universidad Javeriana, Bogotá (Colombia), utilizan las herramientas tecnológicas digitales para apoyar su docencia. La metodología empleada combinó estrategias cualitativas y cuantitativas que posibilitaron la comprensión de los usos que hacen los docentes de las herramientas tecnológicas. Los resultados permitieron delimitar concepciones de tecnología, niveles de acercamiento a las TIC y definición de los trayectos de uso de las TIC en los profesores universitarios. En este artículo se presenta la discusión sobre los trayectos que desarrollan los docentes cuando utilizan las TIC. Las conclusiones muestran las posibilidades que ofrece la cartografía de las trayectorias descritas.

\section{Palabras clave descriptor}

Enseñanza superior, enseñanza, información científica, trayectos de uso.

\section{Transferencia a la práctica}

Los resultados de la investigación contribuyen a la reflexión pedagógica sobre el uso de las tecnologías en la educación superior y a la definición de condiciones de posibilidad para la incorporación de herramientas tecnológicas con sentido en la docencia. Así mismo, a identificar los trayectos de uso que hacen los docentes en la universidad, al visibilizar los usos instrumentales, cognitivos y semióticos atribuidos a la tecnología.

Para citar este artículo / To cite this article / Pour citer cet article / Para citar este artigo Brijaldo-Rodríguez, M. L. \& Sabogal-Modera, M. L. (2015). Trayectos de uso de TIC: Caso de la Universidad Javeriana. magis, Revista Internacional de Investigación en Educación, 7 (15), 135-148. 


\section{Key words plus}

Higher Education, Teaching, Scientific Information, Trajectories of Use.

\section{Transfer to practice}

Results contribute to the pedagogical reflection of the use of technology in higher education and the definition of conditions for incorporating technological tools meaningful in the teaching profession. Also at identifying the paths of use made by university professors, calling attention to the instrumental, cognitive and semiotic uses attributed to technology.

\section{Mots clés descripteur \\ Enseignement supérieure, \\ enseignement, information scientifique, trajectoires d'usage.}

\section{Résumé}

L'objectif de la recherche a été celui de connaître de quelle manière les enseignants universitaires de la Pontificia Universidad Javeriana à Bogota-Colombie utilisent les outils technologiques digitaux pour appuyer son travail d'enseignement. La méthodologie employée a combinée les stratégies qualitatives et quantitatives qui ont rendu possible la compréhension des usages qui fon les enseignants des outils technologique. Les résultats ont permis de faire une délimitation des conceptions de technologie, les niveaux de rapprochement aux TIC et la définition des trajectoires d'usage des TIC dans les enseigants universitaires. Dans cet article on présente le débat sur les trajectoires aui ont développées les enseignants lorsqu'ils utilisent les TIC; les conclusions montrent les possibilités qui offre la cartographie des trajectoires décrites.

\section{Transfert à la pratique}

Les résultats de la recherche contribuent à la réflexion pédagogique de l'usage des technologies dans l'éducation supérieure et la définition des conditions de possibilité pour l'incorporation des outils technologiques avec le sens de l'enseignement. Par ailleurs on identifie les trajectoires d'usage que les enseignants font à l'université et on montre les usages d'outils, cognitifs et sémiotiques attribués à la technologie.
Palavras-chave descritor Ensino superior, ensino, informação científica, trajetos de uso.

\section{Resumo}

O propósito da pesquisa foi conhecer de que maneira os docentes universitários da Pontifícia Universidade Javeriana, Bogotá- Colômbia, utilizam as ferramentas tecnológicas digitais para apoiar sua docência. A metodologia empregada combinou estratégias qualitativas e quantitativas que possibilitaram o entendimento dos usos que fazem os docentes das ferramentas tecnológicas. Os resultados permitiram delimitar concepções de tecnologia, níveis de aproximação às TIC e definição dos trajetos de uso das TIC nos professores universitários. Neste artigo apresenta-se a discussão sobre os trajetos que desenvolvem os do centes quando utilizam as TIC. As conclusões mostram as possibilidades que oferece a cartografia das trajetórias descritas.

\section{Transferência à prática}

Os resultados da pesquisa contribuem com a reflexão pedagógica do uso das tecnologias na educação superior e com a definição de condições de possibilidade para a incorporação de ferramentas tecnológicas com sentido na docência. Também contribuem a identificar os trajetos de uso que fazem os docentes na universidade visibilizando os usos instrumentais, cognitivos e semióticos atribuídos à tecnologia. 
"Cada mapa es una redistribución de callejones sin salida y de brechas, de umbrales y de cercados, que va necesariamente de abajo a arriba."

Gilles Deleuze

\section{Introducción}

Esta investigación tiene como antecedentes los trabajos desarrollados en capacitación y proyectos de virtualización durante cuatro años en el Centro de Educación Asistida por Nuevas Tecnologías ${ }^{1}$ que constituyó la Universidad Javeriana y en el cual se tuvieron dos frentes de acción muy importantes para el desarrollo de la docencia universitaria entre los años 2004 y 2008: el diseño y puesta en marcha de proyectos de virtualización y la formación del docente javeriano en el uso de tecnologías de información y comunicación (TIC). El grupo de investigación Educación y Cibercultura surge a partir de los dos frentes mencionados y realiza la propuesta formativa. Para el desarrollo de proyectos de virtualización se empleó una metodología de acompañamiento en el diseño de la propuesta, la ejecución, puesta en marcha y evaluación del proceso; esta experiencia permitió que los profesores interesados se enfocaran en el diseño de los contenidos y actividades virtuales, mientras un equipo de pedagogos y diseñadores realizaban el montaje de los elementos que conformaban su idea de formación. En cuanto a los procesos de formación del docente javeriano, se desarrollaron y ejecutaron propuestas en temáticas como "Creación de cursos en Uvirtual (Blackboard)", "Docencia virtual", "Diseño de Ambientes Virtuales de Aprendizaje" (AVA) y el "Diplomado de proyectos de virtualización". A partir de estos procesos se observó la necesidad de evaluar tanto las herramientas tecnológicas utilizadas por los docentes como la manera en que se realizaba la incorporación de las TIC en las clases, para analizar las necesidades tecnológicas en la Universidad y su desarrollo en el mediano plazo. Adicionalmente, se veía como campo interesante la reflexión pedagógica en torno al uso de tecnologías por parte de los profesores y así precisar si es posible trazar trayectorias de incorporación de tecnología.

En la literatura revisada se incorporó la investigación desarrollada por César Coll, Teresa Mauri y Javier Onrubia (2008), en la cual se desarrolla una propuesta para revisar los modelos de incorporación de tecnologías en el aula a partir de las prácticas reales de los maestros con empleo de tecnología.

\section{Método}

La metodología empleada combinó un trabajo cuantitativo y otro de orden cualitativo que permitieron derivar una serie de conclusiones que describen la situación actual de la apropiación de algunas de las herramientas tecnológicas utilizadas en la Javeriana y de otras accesibles a través de la Web. El trabajo cuantitativo se desarrolló mediante la aplicación de una encuesta por medio de la cual se indagó por los conocimientos de los docentes acerca de una serie de herramientas tecnológicas que pueden ser utilizadas como recursos para apoyar su docencia, bien sea presencial, virtual o mixta (blended).

1 Grupo de trabajo interdisciplinario conformado por ingenieros, diseñadores gráficos y pedagogos que abordó las dos áreas mencionadas, cuya intervención después de varios años se consolidó en el interés del actual grupo de investigación.

\footnotetext{
Descripción del artículo | Article description | Description de l'article | Artigo descrição

Este artículo de investigación se deriva del proyecto "Usos y construcción de sentido en la implementación de Blackboard y de las otras herramientas de PUJ y los grados de afectación en la comunidad académica", financiado por la Pontificia Universidad Javeriana, Bogotá, en alianza con el Banco Santander, en la convocatoria para proyectos de educación virtual de 2007. En esta se presentó el proyecto del grupo Educación y Cibercultura de la Pontificia Universidad Javeriana. En el estudio participaron cuatro profesoras de la Facultad de Educación: Mónica Ilanda Brijaldo Rodríguez, Nohora Elsa Rodríguez Peña, Martha Leonor Sabogal Modera y Clara Stella Sierra Ávila.

La investigación parte de la pregunta por conocer de qué manera los docentes universitarios javerianos, dadas unas condiciones institucionales y de capacitación ofrecidas por la Vicerrectoría Académica de la Universidad, utilizan las herramientas tecnológicas digitales para apoyar su docencia. La investigación pretendía conocer cómo los docentes de la Universidad se acercan a las herramientas y cómo, a través de dicho acercamiento y del uso que hacen de las herramientas, se trazan unas trayectorias que determinan la manera como se concibe la herramienta y la forma como esta incide en las prácticas docentes.
} 
La encuesta se diseñó a partir de cinco elementos que se consideraron pertinentes para la Universidad en ese momento: 1) datos generales de población; 2) accesibilidad de recursos tecnológicos; 3) capacitación en educación virtual; 4) uso de herramientas tecnológicas; y 5) uso de Blackboard. El procedimiento a seguir para la construcción del cuestionario fue: revisión de encuestas modelo que tuvieran relación con las mismas variables contempladas en el estudio; diseño del cuestionario; selección de la codificación de las preguntas cerradas; aplicación de un piloto, después del cual se realizaron algunos ajustes; y preparación del cuestionario definitivo.

A la encuesta se invitó a 1.800 docentes de la Universidad vinculados de planta y de hora cátedra, que habían participado en procesos de capacitación en educación virtual desde el año 2004 hasta abril de 2008. De ellos se logró respuesta válida a 314 formularios (61,34\% de profesores de planta y $21,7 \%$ de profesores de cátedra). La invitación se hizo mediante el correo electrónico, y se recolectó la información en una base de datos diseñada para este fin. Posteriormente, se organizó y procesó la información recogida, de manera que se pudiera consolidar la mirada general sobre las respuestas obtenidas y los resultados, en términos de conocimiento de herramientas tecnológicas, frecuencia, finalidad y destreza en uso de las mismas. La población de docentes que respondió la encuesta correspondió a un rango de edad entre los 41 y 50 años (32,9\%), seguido por los mayores de 50 (31\%). El 47,6\% pertenece al género femenino y el 52,39\% al género masculino. En el caso de la formación académica, se observó que la mayoría de profesores participantes tiene formación de maestría $(56,1 \%)$, seguida por los estudios de especialización $(17,8 \%)$ y de doctorado $(12,1 \%)$.

Las herramientas indagadas estuvieron conformadas por aquellas que se promueven dentro de la Universidad, como son Blackboard², WikiJaveriana $^{3}$, Cmap Tools, Multiblogs ${ }^{4}$, Wimba Classroom ${ }^{5}$ y Plantilla AVA ${ }^{6}$. Para el caso de las herramientas software social, se tomaron 42 herramientas que contemplan las más comunes en el trabajo con audio, video, redes sociales, representación del conocimiento, comunicación sincrónica, entre otras.

Con el fin de confrontar y ampliar los datos obtenidos en la encuesta, se recurrió al conversatorio, como estrategia para recolectar los datos a través de preguntas abiertas, previamente organizadas, en las que los informantes dieran a conocer las maneras como utilizan las herramientas para apoyar su docencia e interactuar con sus estudiantes. Se realizaron dos eventos, uno presencial y otro de carácter virtual, con población diferente, los cuales permitieron obtener un corpus constituido por las afirmaciones de los informantes en cuanto a inconvenientes, logros y ganancias en el uso de las herramientas en su quehacer docente. Las preguntas desarrolladas en el conversatorio fueron:

2 Es una plataforma computacional flexible, integral y de fácil manejo, que permite la administración de cursos para el aprendizaje. Ofrece herramientas para la interacción sincrónica y asincrónica, la publicación de contenidos y posibilita la administración académica por parte de profesores.

3 Definida en su momento como una plataforma de aplicación tecnológica tipo Sistema de Gestión de Contenidos o CMS (Content Management System), se estructura como un procesador de texto en línea que permite que cualquier usuario registrado pueda escribir e incorporar diferentes elementos, como fotografías, videos, archivos o enlaces de manera sencilla.

4 Generador de Blogs.

5 Software que facilita el encuentro no concurrente de un grupo de personas de forma sincrónica y permite la integración de componentes interactivos, tales como imagen, audio y texto.

6 Es una interfaz gráfica para facilitar el proceso de montaje de contenidos multimedia (texto, animación, sonido, video e imagen), lo que permite la personalización del menú, de la imagen de fondo y del audio. 
1. ¿Qué criterios tiene en cuenta para incorporar herramientas de software social en el aula de clase?

2. ¿Qué significa para usted hacer uso personal y uso académico de las herramientas digitales?

3. ¿Qué finalidad de uso les asigna a las diferentes áreas de la plataforma Blackboard para sus cursos?

4. ¿Qué elementos de diseño tiene en cuenta para crear cursos en Blackboard?

5. ¿Cuáles son las causas para no emplear herramientas de software social, a pesar de conocerlas?

6. ¿Cuáles son las causas para no emplear las herramientas digitales disponibles en la Pontificia Universidad Javeriana, a pesar de conocerlas?

Se contó con la participación de 20 personas en el evento virtual y 10 en el evento presencial. La selección de los participantes se dio en dos órdenes: para el presencial se tuvo en cuenta a aquellos profesores que utilizaban herramientas de software social, y en el virtual, la invitación fue de carácter abierto, para contar con mayor diversidad en las experiencias educativas.

Se recurrió al análisis de contenido, entendido como un enfoque textual que trabaja con procedimientos interpretativos de productos comunicativos (como mensajes, textos o discursos) previamente registrados y basados en técnicas de medida - a veces cuantitativas (estadísticas basadas en el recuento de unidades), a veces cualitativas (lógicas basadas en la combinación de categorías)-, cuyo objeto es elaborar y procesar datos relevantes sobre las condiciones mismas en que se han producido aquellos textos o sobre las condiciones que puedan darse para su empleo posterior.

Según Krippendorff (1990, p. 28), esta técnica sitúa al investigador en una triple perspectiva:

1. De los datos, tal y como se comunican al analista.

2. De contexto de los datos.

3. De la forma como el conocimiento del analista obliga a dividir la realidad.

Esta metodología consta de 10 fases, desarrolladas en su totalidad para la presente investigación:

Fase 1. Determinación de objetivos: caracterizar y evaluar las condiciones de incorporación de las TIC a partir del uso académico (pedagógico y didáctico) de las herramientas tecnológicas que utilizan los docentes javerianos.

Fase 2. Definición del universo a estudiar: docentes de la Pontificia Universidad Javeriana, Bogotá (PUJ), que tienen conocimiento y uso de herramientas tecnológicas en sus clases.

Fase 3. Elección de documentos: el cuerpo de unidades de contenido seleccionado comprendió nociones de TIC, interactividad, niveles de acercamiento a las TIC, ambiente de enseñanza-aprendizaje, tipologías de uso y trayectos de uso.

Fase 4. Definición de fines centrales de la investigación:

a. Caracterizar las condiciones de incorporación de las TIC en las prácticas de los docentes de la PUJ.

b. Tipificar las trayectorias de uso que los docentes hacen de las herramientas tecnológicas y correlacionarlas con las condiciones de incorporación de las TIC. 
c. Interpretar los sentidos que los docentes atribuyen al uso de las TIC en su práctica pedagógica.

d. Aportar elementos conceptuales y prácticos a la reflexión académica y administrativa, para fortalecer el uso y apropiación de las herramientas virtuales en la PUJ por parte de docentes y estudiantes.

Fase 5. Elaboración de indicadores o definición de unidades de análisis:

a. Unidades genéricas: miden la frecuencia de los conceptos definidos.

b. Unidades de contexto: captan el significado de la unidad de registro.

c. Unidades de registro: hacen referencia a una categoría y tienen como finalidad la codificación y el recuento frecuencial.

Fase 6. Reglas de numeración o recuento: forma de contar las unidades de registro codificadas. En este caso, para la investigación se tuvieron en cuenta las siguientes reglas: presencia o ausencia del código, frecuencia, intensidad, contingencia y distribución de frecuencias.

Fase 7. Categorización: en la que se clasifican los elementos de un conjunto a partir de ciertos criterios previamente definidos.

a. Clasificación de las unidades de significado asignado a cada unidad de registro.

b. Codificación como asignación de códigos a cada categoría.

c. Inventario en el que se toman las unidades de significado (codificación) y se da contenido a las categorías.

Fase 8. Exploración del material: se desarrolló a partir de la identificación de unidades de registro, de acuerdo con: los criterios establecidos, la asociación del significado de las unidades de registro con las unidades de significado de valor o antivalor clasificadas en el inventario y la asignación del código de la categoría correspondiente a las unidades de registro.

Fase 9. Fiabilidad y validez: la fiabilidad se estimó calculando el porcentaje de veces que varios codificadores independientes coinciden cuando codifican el mismo material. Se valoró que efectivamente las categorías reunían las características de exhaustividad, pertinencia, objetividad y fidelidad.

Fase 10. Reducción de los datos: en esta fase se llevaron a cabo las operaciones estadísticas, la síntesis y la selección de resultados, para realizar las interpretaciones que dieron lugar a las conclusiones.

Las categorías y subcategorías con las cuales se realizó el análisis de contenido son:

Nociones de TIC: constituyen herramientas o instrumentos mediadores de la actividad mental constructiva de los alumnos y de los procesos de enseñanza, como medio de representación y comunicación novedoso, cuyo uso puede introducir modificaciones importantes en determinados aspectos del funcionamiento psicológico de las personas.

Interactividad: se concreta en determinadas "'formas de organización de la actividad conjunta' de los participantes, que son formatos regulares y reconocibles en torno a los que profesor y alumnos articulan sus actuaciones de acuerdo con unas determinadas reglas o estructuras de participación" (Erickson, citado por Coll et al., 2008, p. 32) social y académica.

Niveles de acercamiento a las TIC: formas de aproximación a las tecnologías cuando estas se asocian a espacios formativos: implementación, incorporación, apropiación. 
Ambientes de enseñanza-aprendizaje: los constituye cualquier combinación a distancia y/o presencial de interacciones de aprendizaje que contenga algún tipo de virtualidad en el tiempo y en el espacio. En esta caracterización, un AVA no comporta indispensablemente la no presencialidad, sino la apropiación de las TIC en el entorno de aprendizaje con intencionalidades pedagógicas.

Tipologías de uso: según Coll y Monereo (2008, p. 4), las tipologías de uso se entienden a partir de "la incidencia que los usos de esas herramientas tienen sobre la actividad conjunta de profesores y alumnos donde reside la clave para analizar su impacto sobre la práctica educativa y, por ende, sobre el aprendizaje de los alumnos. Del mismo modo, es en la incidencia de dichos usos sobre la actividad conjunta donde se concretará o no la capacidad de las TIC de transformar y mejorar las prácticas educativas".

Trayecto de uso: hace referencia a la manera como el docente logra que la tecnología pueda convertirse en un agente eficaz que intenta hacer que lo aprendido de una herramienta pueda utilizarse una y otra vez. Las estrategias desarrolladas con la ayuda de TIC no son lineales, sino que tienen un carácter cíclico en el que cada nuevo conjunto de limitaciones planteadas por las herramientas da lugar a nuevas oportunidades de utilizarlas en desarrollos y estrategias futuras (Pea, 2001).

\section{Resultados}

A partir del análisis de los usos que los docentes hacen de las TIC, la investigación presenta, entre otros, los siguientes resultados:

1. Tres concepciones acerca de las TIC:

a. Desde una perspectiva puramente instrumental, se las entiende como artefactos o aparatos que facilitan algunos procesos, pero no se comprende su sentido en la práctica pedagógica ni la manera como ellas afectan la relación pedagógica entre los distintos actores del proceso.

b. Los docentes que se preguntan acerca de los cambios que las TIC generan en la relación entre profesor y estudiante enfatizan en la importancia del acompañamiento permanente y en su incidencia en los niveles de formación.

c. Las TIC se interpretan como diseño, cuyas posibilidades responden a las intencionalidades pedagógicas pensadas por un docente, lo que las aleja de una apreciación instrumental.

2. De acuerdo con las nociones encontradas, tres tipos de acercamiento a las TIC:

a. Implementación: está asociada con el uso instrumental, en la medida en que limita a una única intención con una comunicación unidireccional del docente productor al estudiante receptor. Se adopta la tecnología como una extensión de la clase presencial, regida por el esquema de actividad mental tradicional de transmisión de conocimientos.

b. Incorporación: se concibe el uso de las herramientas como apoyo y complemento de la docencia, y se lo relaciona con el establecimiento de nuevas formas de comunicación y aprendizaje y con una mayor reflexión acerca de los criterios de diseño del profesor. 
c. Apropiación: se analiza el establecimiento de relaciones intersubjetivas, con la intención de generar espacios de construcción conjunta y prácticas que conllevan aprendizajes más planeados, organizados y reflexivos, que no dependen solo de la tecnología, sino que la resignifican y la reconstruyen a partir de las necesidades de la docencia.

3. Con base en las concepciones y tipos de acercamiento a las TIC, se pueden definir los trayectos de uso que hacen los docentes de ellas. Estas trayectorias se pueden describir cuando el docente deconstruye la herramienta tecnológica, los niveles de interacción y la pertinencia de la herramienta. A continuación, se realiza la discusión sobre el tema de los trayectos.

El tema de los trayectos en las TIC tiene especial relevancia en los resultados de la presente investigación, puesto que reconstruyen los pasos que recorre un docente desde el momento que decide hacer uso de las TIC como posibles mediaciones del proceso de enseñanza-aprendizaje. Los recorridos por las TIC que establece cualquier profesor tienen la particularidad de ser trazados según parámetros generalizables -para la mayoría de ellos-y permiten diseñar mapas con puntos en común, hasta cuando la trayectoria de los mismos se bifurca en diferentes caminos.

Los trayectos de uso de las TIC se pueden considerar como la construcción de una cartografía subjetiva, trazada por quienes recorren el mapa como un medio de llegar a algún lado, que de igual manera refleja a quien lo recorre. En palabras de Deleuze (1997, p. 81), en ellos se "expresa la identidad del itinerario y de lo recorrido".

Un trayecto se establece a partir de cualidades, de acontecimientos, de sustancias y de fuerzas. Así, los trazos que marca un individuo cualquiera mantienen una estrecha relación entre lo real y lo imaginario o subjetivo y desarrollan un entramado de recorridos que permiten a los mapas superponerse, "de tal modo que cada cual encuentra un retoque en el siguiente, en vez de un origen en los anteriores: de un mapa a otro, no se trata de la búsqueda de un origen, sino de una evaluación de los desplazamientos" (p. 82). Cuando los recorridos se establecen en mapas, dejan de ser parte de los sujetos o los medios y se convierten en trayectos y devenires. Los recorridos son en esencia un inconsciente de movilización, a los cuales se les puede trazar su trayectoria e identificar los posibles indicadores de nuevos devenires.

Es así como los trayectos de uso de las TIC se pueden trazar a partir de las trayectorias y los devenires que cada docente recorre cuando inicia una trayectoria en el uso de TIC. A continuación se mostrarán los entramados que se identificaron en el desarrollo del proyecto.

La categoría de trayectos se planteó desde el inicio del proyecto y hace referencia a la manera como los usuarios de la tecnología, en general, aprenden el uso de una herramienta determinada y la convierten en un "artefacto transparente". Como comenta Gui Bonsiepe (1993), el profesor ya no tiene necesidad de pensar en el diseño del programa o herramienta y el estudiante puede ocuparse de la realización de la tarea que se propone ejecutar. Para De Kerckhove (1999), todas las tecnologías tienen dos fases; en la primera el dispositivo es evidente y en la segunda se lo interioriza, hasta convertirse en invisible.

Las maneras como el docente se relaciona con las herramientas pueden comportar diversas miradas, dependiendo del uso que se les otorgue a las mismas. En el momento en que un docente logra interiorizar una herramienta tecnológica, se dice que le asigna un sentido a la misma, es decir, que establece una utilidad particular que le permite diseñar las estrategias de uso que orientarán el proceso de enseñanza-aprendizaje y los conocimientos que espera lograr en sus estudiantes.

Otro aspecto relevante es el de la inteligencia distribuida, que Pea (1993, p. 76) caracteriza como "la construcción social del conocimiento por medio de esfuerzos cooperativos dirigidos a metas compartidas o de diálogos y cuestionamientos que nacen de las diferencias que existen entre las experiencias de las personas". Esta inteligencia distribuida puede estar presente en los diversos artefactos que han sido diseñados para la realización de tareas complejas, entre los que se encuentran las interfaces de los programas de computador. El mismo autor afirma que las estructuras mediadoras de la actividad abarcan los objetos diseñados e incluyen a las personas en sus relaciones sociales y el entorno físico, el cual tiene relación directa con los deseos del ser humano, siendo estos últimos las instancias que presionan la significación y resignificación del entorno de personal, incluidos los usos de las TIC para el diseño y ejecución de acciones de enseñanza y aprendizaje.

En una segunda instancia estaría el significado de uso, que se da cuando el docente es capaz de formular su propuesta formativa pensando en el estudiante, en la interacción de la herramienta, en el modelo mental del docente al hacer uso de las herramientas y en la situación real de interacción que se logra. Esto lleva a que el docente evalúe las posibilidades que le ofrece la herramienta y, a partir de las realidades educativas, plantee la posibilidad de continuar o abandonar el uso de una herramienta.

Y para terminar, se contempla el trayecto de uso, que hace referencia a la manera como el docente 
logra que la tecnología pueda convertirse en una herramienta de enseñanza eficaz e intenta hacer que lo aprendido de esta, pueda utilizarse una y otra vez. Como se dijo, las estrategias desarrolladas con la ayuda de TIC no son lineales, sino que tienen un carácter cíclico en el que cada nuevo conjunto de limitaciones planteadas por las herramientas da lugar a nuevas oportunidades de utilizarlas en desarrollos y estrategias futuras (Pea, 2000).

En cuanto a las limitaciones del estudio, estuvieron relacionadas con el enfoque local, por cuanto se tuvo en cuenta únicamente la población de docentes participantes en los eventos de formación en tecnologías de la Pontificia Universidad Javeriana, y aunque esta es una muestra válida como estudio de caso, sería importante poder establecer la comparación con otras instituciones de educación superior del país. Así mismo, el tipo de herramientas tecnológicas analizadas se restringió a las usadas y adaptadas por los profesores de la Javeriana.

\section{Sentido de uso de las TIC}

El sentido de uso se define como la reflexión acerca de las estrategias, métodos y metodologías que orientarán los procesos de enseñanza-aprendizaje en el contexto donde se interactúa y las posibilidades de una u otra tecnología según la concepción del sistema educativo. El sentido se corresponde con las maneras de apropiación de las tecnologías e implica reflexionar acerca de la configuración de entornos desde perspectivas pedagógicas y didácticas y la selección de diferentes herramientas para la enseñanza cognitiva.

La reflexión pedagógica que realiza el docente le permite: establecer las estrategias de uso de las TIC, al igual que la manera como logra interiorizar tanto la interfaz ${ }^{7}$ como la arquitectura de la herramienta; el paso del uso personal al uso académico o el uso exclusivamente académico de una herramienta determinada; y el diseño de ambientes de aprendizaje y el apoyo a la docencia mediante diversos tipos de tecnología.

Significado de uso de las TIC

El significado de uso de las TIC se define como la propuesta formativa realizada por el profesor, desde enfoques paradigmáticos, que corresponde con maneras de entender nociones como: conocer, enseñar,

7 Interfaz, de acuerdo con Scolari (2004), son espacios conceptuales de deconstrucción en los que la percepción del usuario adapta y acondiciona las herramientas tecnológicas de acuerdo con los propios usos y las necesidades de ampliar o modificar su utilidad. Igualmente, incluye la relación dialógica entre el usuario y el diseñador, en cuanto al diseño de la interfaz y de las interacciones que se dan en el proceso de diseño. aprender, interactuar y evaluar, que van a determinar el sentido de la incorporación de las tecnologías.

El docente, desde y en la interacción, decide utilizar apoyos y recursos necesarios para poder conectar con la representación del contenido del alumno y modificarla en función de la representación que desea construir. De esta manera, se van compartiendo significados cada vez mayores, específicos y cercanos a lo que culturalmente se acepta para el contenido a ser trabajado.

El significado se entiende, entonces, como el paso posterior al sentido, en el cual el docente formula su proceso de enseñanza-aprendizaje mediado por herramientas y establece el objetivo de uso para su espacio educativo. Igualmente, comprende las modificaciones y transformaciones que suceden en el uso de las herramientas y cómo se logran compartir las experiencias y el aprendizaje colectivo de las TIC. El significado se relaciona con las posibilidades de transformación y el cambio de paradigmas a las que se ve abocado el docente cuando incorpora tecnologías en sus prácticas, lo que conlleva a la resignificación de su quehacer y el uso académico y no académico de las herramientas.

\section{Trayectorias de uso}

Son los espacios conceptuales de deconstrucción en los cuales la percepción del docente suele adaptar y acondicionar las herramientas tecnológicas de acuerdo con los propios usos y las necesidades de ampliar o modificar su utilidad. La construcción de trayectorias se da en la interactividad, el profesor ajusta la ayuda y progresivamente logra compartir con el estudiante niveles mayores de interacción. Este proceso de negociación es posible gracias al lenguaje, ya que permite representar de maneras distintas los objetos, acciones y acontecimientos y posibilita diversos grados de intersubjetividad desde donde transitar de contextos sociales de referencia a contextos específicos de referencia.

Las trayectorias de uso de las TIC de los docentes de la Pontificia Universidad Javeriana se conciben como espacios conceptuales que permiten el desarrollo, la adaptación y el acondicionamiento de las herramientas según las necesidades propias de cada profesor. Las herramientas se deconstruyen según el conocimiento de las mismas, los niveles de interacción que se logran con los estudiantes, el desarrollo de trabajos colaborativos, la reflexión en torno a si es la herramienta adecuada para el proceso de aprendizaje que se planteó y las transformaciones que se logran en el aprendizaje.

Las trayectorias están en relación con las herramientas de enseñanza semiótica en el diseño y representación, con los procesos de reflexión y análisis acerca de las tecnologías, con el fin de apropiarlas y 
adaptarlas a sus intereses y necesidades. Obedece al grado de dominio que se logra de una herramienta, cuando se ha superado la preocupación por el funcionamiento de la misma y se comparten los significados asignados a las herramientas tecnológicas.

Mapas de trayecto de los docentes

El sentido de uso de las herramientas tecnológicas utilizadas por los profesores de la Pontificia Universidad Javeriana se produce mediante la selección de tecnologías que tiene como ejes la arquitectura, la interfaz y el posible uso académico de la herramienta. Si estos ejes se ajustan a las necesidades del docente, las herramientas logran la "significación". El significado de uso de una herramienta tecnológica se logra cuando se resignifica el quehacer académico del docente y cuando se apropia la herramienta como una mediación pedagógica en el proceso de enseñanza-aprendizaje.

Para esta investigación, con la resignificación de las herramientas de software social estudiadas, en la mayoría de los casos, estas se convierten en espacios para la interacción y la comunicación entre docentes y estudiantes y entre los mismos estudiantes, que van desde charlas informales hasta lugares de encuentro académico o de resolución de dudas.

En el caso de las herramientas propias de la Pontificia Universidad Javeriana, el sistema de videoconferencia es la herramienta más usada y se resignifica como herramienta de enseñanza eficaz, en un salón de clase virtual donde se logra constituir espacios de interacción en tiempo real.

En cuanto a la plataforma Blackboard, la resignificación se logra mediante el uso extendido de los servicios de la plataforma y la incorporación de otras herramientas tecnológicas que complementan las funcionalidades preestablecidas en la misma. Es de anotar que la transformación no sucede en la configuración o arquitectura de la plataforma, sino en la manera como el docente incorpora nuevas estrategias educativas a partir de los servicios propios de la herramienta o del uso de otras que lo complementen.

Desde estos significados logrados, las trayectorias de uso se describen cuando el docente deconstruye la herramienta tecnológica a partir del conocimiento y la destreza lograda, del uso continuo de la misma, de los niveles de interacción que se establecen con los estudiantes y de la reflexión sobre la pertinencia de la herramienta para el proceso de aprendizaje planteado.

En los trayectos que se trazan para usar las tecnologías se descubren mapas de trayectos que configuran un entramado de recorridos, que cambian de rumbo según el trayecto que se escoja. Los trayectos que se pueden configurar, en la Pontificia Universidad Javeriana, a partir de las trayectorias de los docentes en el uso de TIC, se delinean como:

- Espacio académico homologable a una clase presencial (donde prima la oralidad), en la cual se recurre al uso de las TIC para suministrar información esencialmente igual a la que se brinda en un espacio presencial

- Espacio para la comunicación e interacción de carácter personal, trayecto en el cual se potencian las herramientas de comunicación para uso fundamentalmente personal, pero no se logra trasladarlas al escenario académico.

- Espacio para la comunicación e interacción académica entre docentes y estudiantes, en el cual sí se ven las posibilidades de comunicación entre docentes y estudiantes, que se amplían mediante el uso de estas herramientas. 
- Espacio para la publicación de contenidos, cuando las herramientas se emplean principalmente como repositorios de contenidos.

- Espacio de ambiente virtual de aprendizaje, en el cual se reflexiona acerca de la manera como se pueden diseñar ambientes de aprendizaje mediados por tecnologías digitales.

La representación de estos trayectos se presenta a continuación (véase esquema de la Figura 1) y su intención es hacer un ejercicio cartográfico que permita visualizar la composición de las trayectorias que se encontraron en la presente investigación.

Figura 1

Esquema del trayecto de uso de TIC por los docentes universitarios

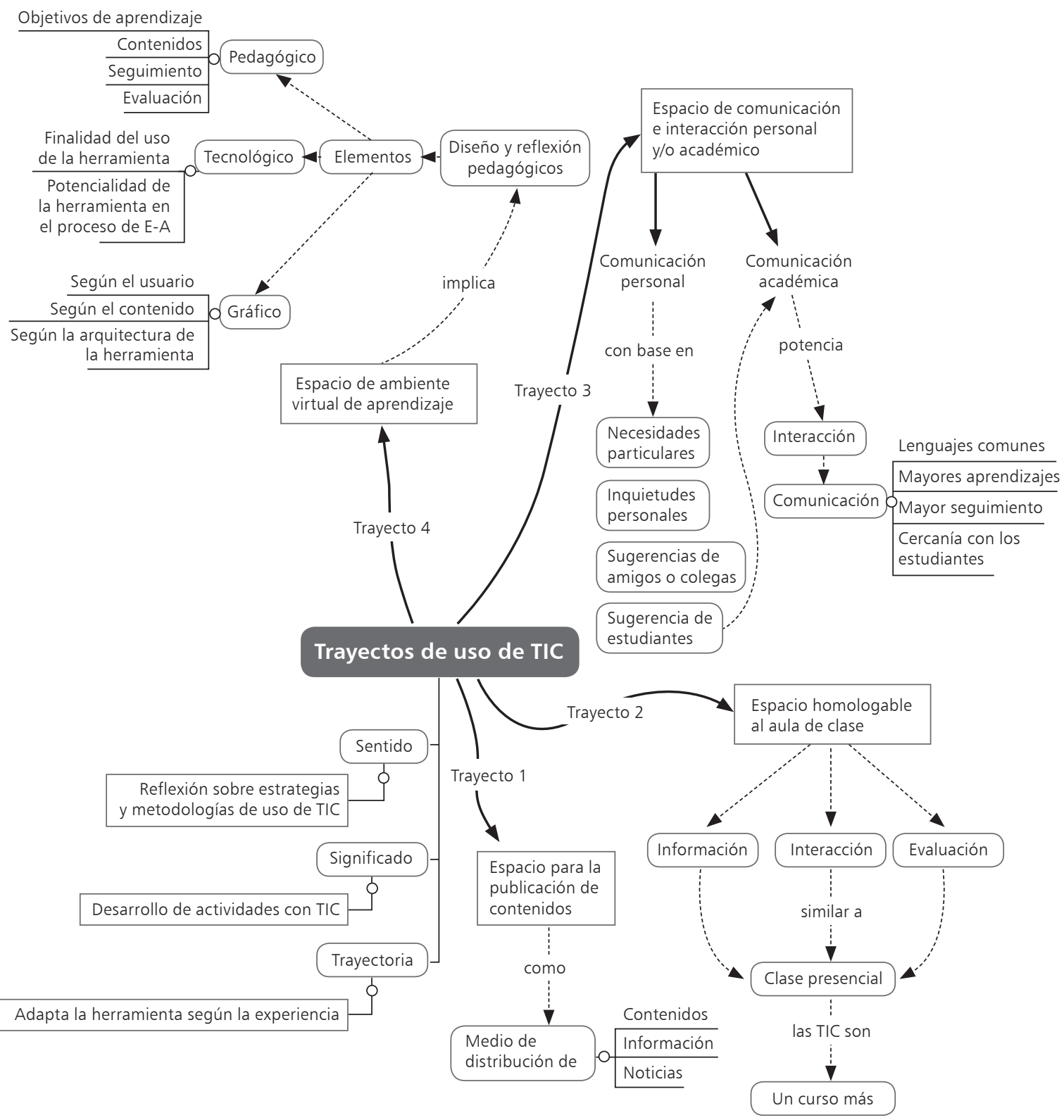

Fuente: Elaboración propia 
Trayecto 1: Espacio para la publicación de contenidos

En este trayecto se observa que los docentes realizan la reflexión sobre el sentido de la herramienta y la significan como un medio para distribuir y almacenar contenidos, información de carácter general del curso y espacio de divulgación de noticias o anuncios. La adaptación que se configura en esta trayectoria es la de un administrador de contenidos. El docente solamente la usa para tener disponibles los documentos y materiales del curso o cursos para los estudiantes.

\section{Trayecto 2: Espacio homologable al aula de clase}

Para este trazado el docente plantea como metodología de enseñanza la simulación del salón de clase presencial; por tanto, utiliza las herramientas como medios para distribuir la información y realizar la interacción con los estudiantes y en algunos procesos de evaluación (por ejemplo, el registro de entrada a la clase), de modo que emula por completo el desarrollo del curso a la manera como se realiza un clase presencial, solo que en este caso se emplean herramientas tecnológicas que permitan los encuentros sincrónicos -como en los sistemas de videoconferencia Horizon Wimba o Elluminate- $u$ otras que hacen posible controlar el ingreso y el desarrollo de actividades -como en la plataforma Blackboard-. En este trayecto, las herramientas se significan como sistemas de control de actividades, que además privilegian la interacción oral entre docente y estudiante, pero no entre estudiantes o entre estudiante y docente.

Trayecto 3: Espacio de comunicación e interacción personal $y / 0$ académico

En este trayecto la reflexión sobre las estrategias para el uso de las herramientas se conforma a partir de mecanismos de comunicación, bien sea de carácter personal o de carácter académico. En este caso, se entiende la comunicación personal como los espacios personales de conversación, las inquietudes particulares del docente y la charla informal con los estudiantes. La comunicación académica hace referencia a las posibilidades de utilizar herramientas digitales que permitan la comunicación instantánea, la resolución de dudas inmediatas, la posibilidad de generar discusiones en línea y el manejo de lenguajes visuales y sonoros, además de la conversación por escrito (Messenger o chat) o en audioconferencia (Messenger, Skype).

La significación de las TIC en el trayecto 3 se logra a partir de las necesidades del docente de establecer lugares de encuentro, y la trayectoria se marca en tres rutas: una que puede surgir y permanecer como comunicación de carácter personal, otra que surge en la comunicación personal y se transforma a comunicación académica (muchas veces por iniciativa de los estudiantes) y la que surge desde la comunicación estrictamente académica.

\section{Trayecto 4: Espacio de ambiente virtual de aprendizaje}

En el trayecto 4 se reflexiona de manera pedagógica y se diseñan los ambientes de aprendizaje a partir de tres elementos primordiales: lo pedagógico, lo tecnológico y lo gráfico. Para el elemento pedagógico, se realiza el diseño de la formulación de los objetivos de aprendizaje, los contenidos que se trabajan en el curso y el seguimiento y la evaluación del proceso de aprendizaje del estudiante. Se intuye que la preocupación del docente es eminentemente formativa y que, en ese sentido, busca en las TIC oportunidades de impulsar los aprendizajes de sus estudiantes.

Para el elemento tecnológico se tiene en cuenta la versatilidad y la posibilidad de intervenir la herramienta seleccionada - de acuerdo con la reflexión pedagógica- a partir de la finalidad de uso que el docente le asigna y la evaluación de las potencialidades de la misma en el proceso de enseñanza-aprendizaje. Se ha podido observar que en cuanto la herramienta permite la trasformación de servicios, la programación de otros y, en general, la interacción entre usuarios, y mejores condiciones de uso, estas se resignifican más rápidamente. Esta puede ser una de las razones por las cuales las herramientas de software social o de la web 2.0 han alcanzado relevancia en los ambientes educativos.

Para el elemento gráfico, se analiza la interfaz de la herramienta desde las opciones que presenta al usuario, las posibilidades de alcanzar interactividad en los contenidos, la estructura y la arquitectura, según el criterio de facilidad de manejo de la misma. Este elemento se asocia también con el enriquecimiento audiovisual que ofrece para el aprendizaje de los estudiantes, como en el caso de los cursos de Biología, Artes o Diseño.

La adaptación de la(s) herramienta(s) según la experiencia de uso es el eje del trazado de este trayecto, que es el más complejo y dinámico, y por ello es el recorrido que menos se da entre los docentes, por cuanto requiere mayor reflexión del proceso educativo y de los aportes que conllevan las herramientas.

Dentro de las limitaciones de la investigación está la que presenta la noción de trayectos como construcción teórica que requiere la validación en la práctica del docente; por tanto, es necesario continuar en la consolidación de esta categoría a través del análisis del quehacer docente y del uso pedagógico de las TIC en la educación superior. Otra limitación estuvo referida a las herramientas que se revisaron en la investigación, las cuales se enmarcaron únicamente en la propuesta institucional, dejando de lado herramientas de software libre. 


\section{Conclusiones}

Entre las conclusiones destacables de la investigación, se pueden mencionar las siguientes:

El uso que hacen los docentes de las TIC en la Pontificia Universidad Javeriana se puede cartografiar en "mapas de trayectos", que pueden ser los mismos recorridos y devenires de otros profesores universitarios.

El trazado de los recorridos que hacen los profesores en el uso de herramientas se fundamenta en el sentido que les asignan, en el significado que estas adquieren y en la trayectoria que ellas adoptan según el trabajo realizado con ella. Dichos recorridos pueden tener longitudes diversas y, según sea el grado de desarrollo que les imprima el profesor, unos pueden ser muy puntuales y otros pueden dibujarse a partir de los de otros, haciendo que su recorrido sea más extenso y posiblemente más reflexivo.

Los mapas resultantes del proceso de análisis de los usos de las herramientas son una propuesta de lectura y es probable que los recorridos puedan ir adquiriendo nuevos elementos y aspectos que reconfiguren los trayectos o incluyan nuevos devenires. En este sentido, los trayectos se hacen insospechados, continuos y dinámicos, dependientes de las experiencias de los profesores y de las posibilidades de contacto con las nuevas experiencias de aprendizaje mediadas por herramientas tecnológicas.

El estudio ofrece amplias posibilidades de futuras investigaciones en relación con la identificación de las maneras como los docentes efectivamente incorporan, implementan o apropian las tecnologías de información a sus prácticas educativas, así como las maneras en que las trayectorias se ven reflejadas en los procesos de enseñanza-aprendizaje.

Por último, la realización de este proyecto permitió establecer parámetros de análisis novedosos en cuanto al tipo de grados de apropiación de la tecnología y trayectos de uso por parte de los docentes universitarios. Un nuevo proceso investigativo permitirá la revisión y el análisis de experiencias de prácticas docentes a partir de estos hallazgos, que puedan llevar a la precisión de políticas de enriquecimiento de los ambientes de aprendizaje por medio de tecnologías en la educación superior, en Colombia y en Latinoamérica.

\section{Sobre las autoras}

Mónica Ilanda Brijaldo-Rodríguez es docente de la Facultad de Educación de la Pontificia Universidad Javeriana, candidata a Doctora en Procesos de Formación en Espacios Virtuales, de la Universidad de Salamanca, España. Líder del grupo de investigación en Educación y Cibercultura. Experta en el uso de TIC en el aula y de videojuegos para entornos educativos.

Martha Leonor Sabogal-Modera es docente de la Facultad de Educación de la Pontificia Universidad Javeriana. Actualmente, es directora de la Licenciatura en Educación Básica con Énfasis en Humanidades en Lengua Castellana. Licenciada en Lenguas Modernas y Magister en Educación, de la Universidad Javeriana. Miembro del grupo de investigación en Educación y Cibercultura.

\section{Referencias}

Badia, A., Mauri, T. \& Monereo C. (2004). La práctica psicopedagógica en educación formal. Universitat Uberta de Catalunya - UOC. Recuperado el 24 de junio de 2009 de http://books.google.com.co/books?id=5yMNLWcPavEC $\& d q=u s o+$ instrumental + de+las+TIC\&source $=$ gbs_navlinks_s

Barrera, J. (1998). En torno al sentido -didáctica y hermenéutica-. Revista Colombiana de Humanidades, 63 (enero-diciembre). 
Bates, T. (2000). Cómo gestionar el cambio tecnológico. Estrategias para los responsables de centros universitarios. Barcelona: Gedisa.

Bonsiepe, G. (1993). El papel del diseño. En Giovanni Anceschi (comp.), El proyecto de la interfaz. Milán: Domus Academy.

Coates, T. (2003). My working definition of social software... Recuperado en marzo de 2007 de http://www.plasticbag.org/archives/2003/05/my_working_definition_of_social_software/

Coll, C. (2004). Psicología de la educación y prácticas educativas mediadas por las tecnologías de la información y la comunicación: una mirada constructivista. Sinéctica, 25. Recuperado el 24 de junio de 2009 de http://www. ub.edu/grintie

Coll, C., Majós, T. M. \& Onrubia, J. (2005). Análisis de los usos reales de las TIC en contextos educativos formales: una aproximación socio-cultural. Redie. Revista Electrónica de Investigación Educativa, 10 (1). Recuperado el 8 de agosto de 2014 de http://www.oei.es/noticias/spip.php?article4004

Coll, C. \& Monereo, C. (eds.) (2008). Psicología de la educación virtual. Madrid: Morata.

Coll, C., Onrubia, J. \& Mauri T. (2008). Ayudar a aprender en contextos educativos: el ejercicio de la influencia educativa y el análisis de la enseñanza. Revista de Educación, 346 (mayo-agosto), 33-70.

De Kerckhove, D. (1999). Inteligencias en conexión. Hacia una sociedad de la Web. Barcelona: Gedisa.

Deleuze, G. (1997). Crítica y clínica. Barcelona: Anagrama.

Domínguez Figaredo, D. (2006). Etnografía educativa más allá de la etnografía escolar. Trasvase de significados al ciberespacio. Forum: Qualitative Social Research, 7 (2), art. 2. On-line Journal. Recuperado en marzo de 2007 de http://www.qualitative-research.net/index.php/fqs/article/view/95/199

Dubost, L. (2006). XWiki: Building Applications in a Wiki. ObjectWeb - Annual Conference 2006. Recuperado en marzo de 2007 de http://objectwebcon06.objectweb.org/xwiki/bin/Main/Session

Epper, R. \& Bates, T. (2004). Enseñar al profesorado cómo utilizar la tecnología. Buenas prácticas de instituciones líderes. Barcelona: Universitat Uberta de Catalunya - UOC.

Krippendorf, K. (1990). Metodología de análisis de contenido: teoría y práctica. Barcelona: Paidós.

Pea, R. (1993). Prácticas de inteligencia distribuida y diseños para la educación. En Gavriel Salomón (comp.), Cogniciones distribuidas. Buenos Aires: Amorrortu.

Pea, R. (2001, february). Using information technology to change the way we learn. In S. L. Graham \& A. J. Viterbi (Chairs), Panel on Transforming the Way We Learn (Invited Participant and co-author). Recuperado el 15 de mayo de 2011 de http://www.stanford.edu/ roypea/RoyPDF\%20folder/ A105_PITAC_01.pdf

Pérez, T. (1999). El proyecto de investigación y el diseño metodológico. Cuarto módulo, Serie Investigación, Maestría en Educación. Bogotá: Pontificia Universidad Javeriana.

Picardo, Ó. (2002 julio). Pedagogía informacional: Enseñar a aprender en la sociedad del conocimiento. Universitat Oberta de Catalunya - UOC. Recuperado en marzo de 2007 de http://www.uoc.edu/web/esp/art/uoc/opicardo0602/opicardo0602.html\#3

Ruiz Silva, A. (2004). Texto, testimonio y metatexto. El análisis de contenido en la investigación en educación. En La práctica investigativa en Ciencias Sociales. Bogotá. Universidad Pedagógica Nacional.

Scolari, C. (2004). Hacer clic. Hacia una sociosemiótica de las interacciones digitales. Barcelona: Gedisa. 